\title{
A-FABP-PTEN/AKT Regulates Insulin Resistance in Preadipocyte Cell 3T3-LI Cells
}

\author{
Rensiqin $\mathrm{Wu}^{\prime}$ \\ Hui Wang' \\ Jian Huangfu' \\ Rui Xiao ${ }^{2}$ \\ 'Department of Endocrinology, The \\ Affiliated Hospital of Inner Mongolia \\ Medical University, Hohhot, 010000 , \\ Inner Mongolia, People's Republic of \\ China; ${ }^{2}$ Key Laboratory of Molecular \\ Pathology, Inner Mongolia Medical \\ University, Huhhot, 010000, Inner \\ Mongolia, People's Republic of China
}

Correspondence: Jian Huangfu Department of Endocrinology, The Affiliated Hospital of Inner Mongolia Medical University, NO.Ist Tunnel North Road, Hohhot, 010000, Inner Mongolia,

People's Republic of China

Tel +86-18686053304

Email hfj999dr@yeah.net

Rui Xiao

Key Laboratory of Molecular Pathology, Inner Mongolia Medical University,

NO.Ist Tunnel North Road, Huhhot, 010000, Inner Mongolia, People's Republic of China

Tel +86-18686053304

Email xiaorui79@2Icn.com
Objective: The purpose of this study was to explore the regulation of A-FABP-PTEN/AKT on insulin resistance in preadipocyte 3T3-L1 cell.

Methods: siRNA interference method was used to knock-down the A-FABP expression in 3T3L1 cells. The cell proliferation was detected by oil-O staining and MTT. The protein and mRNA expression levels of A-FABP, PTEN and AKT were detected by Western blot and qPCR.

Results: Inhibition of A-FABP expression increased cell proliferation activity of the 3T3-L1 cells. Moreover, siRNA3 significantly reduced A-FABP mRNA expression compared with siRNA1 and siRNA2 $(P<0.05)$. The A-FABP mRNA level was significantly increased in the induced 3T3-L1 cells, while the PTEN mRNA expression was significantly decreased $(P<0.05)$. Inhibition of A-FABP can significantly increase the PTEN mRNA expression in the process of induced 3T3-L1 cells $(P<0.05)$. Overexpression of A-FABP can also increase the PTEN mRNA expression in the process of 3T3-L1 cell proliferation $(P<0.05)$. Furthermore, the protein expression levels of PTEN and p-AKT expression were not changed in the process of 3T3-L1 cell proliferation with or without A-FABP interference $(P>0.05)$. However, inhibition of A-FABP significantly increased the PTEN protein expression and reduced the p-AKT protein expression in the induced 3T3-L1 cells.

Conclusion: Our finding suggested that A-FABP can directly inhibit the phosphorylation of AKT and increase the PTEN expression in the process of normal adipocyte differentiation, which speculated that A-FABP played a crucial role by adjusting the AKT activity in the process of adipocyte differentiation.

Keywords: A-FABP, PTEN, AKT, preadipocyte differentiation, insulin resistance

\section{Introduction}

As we all known, obesity can lead to insulin resistance. However, patients with normal or even low body mass index $(\mathrm{BMI})(<23.9)$ may develop insulin resistance, type-2 diabetes mellitus (DM) and other metabolic syndromes. Actually, fat is the regulator for energy balance, endocrinology and metabolism. Fat tissues can secrete multiple bioactive substances that also are called adipokines. Previous study has found that compared to the increase of the simple adipocyte volume, abnormalities in adipocyte proliferation are more closely associated with insulin resistance. ${ }^{1}$ Abnormalities in both proliferation and differentiation of preadipocytes lead to excessive fat accumulation and consequently cause endocrine dysfunction, which results in the development of insulin resistance and type- $2 \mathrm{DM}^{2}$

Adipocyte fatty acid-binding protein (A-FABP), a member of the family of fatty acid-binding proteins (FABPs), is discovered in adipocytes. A-FABP is mainly expressed in adipocytes and macrophages. As the transporter of fatty acid, A-FABP 
influences the metabolism of fatty acid, and it also enters cell nucleus to affect the transcription activity of peroxisome proliferator-activated receptor $\gamma$ (PPAR- $\gamma$ ) ${ }^{3}$ PPARg is one of the therapeutic targets for antifibrotic therapy. ${ }^{4}$ A previous study has revealed that A-FABP-/- mice cannot resist obesity induced by high-fat diet, but they are able to obviously overcome hyperinsulinism and insulin resistance induced by obesity. ${ }^{5,6} \mathrm{Xu}$ et al have analyzed serum A-FABP concentration in 229 men and women of different age by tandem mass spectrometry, which indicates that serum A-FABP level is positively correlated with waistline, blood pressure, dyslipidemia, fasted serum insulin and IR. ${ }^{7}$

Phosphatase and tensin homolog deleted on chromosome ten (PTEN) is the first cancer suppressor gene with phosphatase activity so far. ${ }^{8}$ A novel study has showed that PTEN has interaction with A-FABP in predifferentiation preadipocyte 3T3-L1 cells. ${ }^{9}$ Furthermore, a gene expression analysis on the PTEN deletion in keratinocytes displays that the A-FABP expression is increased by 5 folds after PTEN deletion. ${ }^{10}$ Yuan-yuan LI et al also has found that patients with DM have more severe insulin resistance than healthy controls, partially contributed by elevated plasma levels of FABP4 and PTEN. ${ }^{11}$ The gene expression levels of PTEN and FABP4 in the liver, muscle, and adipose tissues in T2DM rats were all significantly higher than those in the control rats. ${ }^{12}$ PTEN can act as a lipid phosphatase to negatively regulate PI3K/AKT path. It is seen that PTEN inhibits proliferation, survival and growth of cells as well as interferes in glucose metabolism by blocking P13K-AKT signaling pathway. ${ }^{13}$

Increased differentiation of adipocytes may lead to added number of mature adipocytes and subsequently contribute a certain part to the development of obesity. ${ }^{14,15}$ Therefore, the effects of A-FABP on proliferation and differentiation of preadipocytes are also able to microcosmically offer theoretical basis for the role of A-FABP in adipogenesis process. Therefore, in the present study, we aimed to explore the regulation of A-FABP-PTEN/AKT on insulin resistance in preadipocyte 3T3-L1 cells.

\section{Materials and Methods}

\section{Materials}

Mouse adipose precursor 3T3L1 cells were obtained from Shanghai Institute of Biochemistry and Cell Biology, Chinese Academy of Sciences (Shanghai, China). Dulbecco's modified eagle's medium (DMEM) was purchased from (Gibco, USA). Fetal bovine serum (FBS) was purchased from (Hyclone). Insulin, dexamethasone, 3 isobutyl 1 methylxanthine (IBMX) and oil red O staining were purchased from (Sigma, USA). The required antibodies, including anti-A-FABP4, anti-PTEN, anti-p-AKT and anti- $\beta$-actin, were purchased from (Cell Signaling). Diethyl pyrocarbonate (DEPC) was purchased from (Amresco). SYBRGreen PCR kit was purchased from ABI.

\section{Cell Culture}

3T3L1 cells were cultured in DMEM supplemented with cyanine and streptomycin (final concentration $100 \mathrm{U} / \mathrm{mL}$ ) and $10 \%$ FBS. The cells were cultured in an incubator at $37{ }^{\circ} \mathrm{C}$ and a $5 \% \mathrm{CO}_{2}$ concentration. The medium was changed every other day. When the degree of cell confluence reached $80-90 \%$, it was digested with $0.25 \%$ trypsin for subculture.

\section{Oil Red O Staining}

The 3T3L1 cells were cultured for $48 \mathrm{~h}$, DMEM with $15 \%$ FBS, insulin and dexamethasone (American sigma company) were added to. The 3T3L1 cells were cultured for another $48 \mathrm{~h}$, the medium was changed to DMEM with $15 \%$ FBS. The 3T3-L1 cells differentiated for 10-12 days were mostly fat cell phenotypes. The fat cells were then identified by oil red $\mathrm{O}$ dye (American sigma). Briefly, cells were fixed in $10 \%$ neutral buffered formalin solution for $30 \mathrm{~min}$ at room temperature and washed twice with PBS. Filtered oil red $\mathrm{O}$ dye was added and incubated at room temperature for $60 \mathrm{~min}$. Then, the oil red $\mathrm{O}$ dye was absorbed and discarded. The effluent was washed with running water until it was colorless and clear, and it was observed and photographed under the microscope.

\section{Small Interfering RNA (siRNA)}

In this study, three siRNAs were used to inhibit the expression of A-FABP mRNA. siRNA was transfected with lipofectamine ${ }^{\mathrm{TM}} 2000$ (Invitrogen). 3T3-L1 cells were seeded on a 6 -well plate $\left(1 \times 10^{5}\right.$ cells/well $)$. When the cell density reached $60 \%$, the medium was removed and replaced with serum-free and antibiotic-free medium. Then, $500 \mu \mathrm{L}$ opti-MEM containing $10 \mu \mathrm{L}$ lipofectamine $^{\mathrm{TM}} 2000$ was added. After mixing, the cells were cultured in $5 \% \mathrm{CO}_{2}$ at $37{ }^{\circ} \mathrm{C}$ for $6 \mathrm{~h}$. The culture medium was discarded and normal DMEM (containing 10\% FBS) was added to culture for further experiments. 


\section{RNA Isolation and Real-Time PCR}

Total RNA was extracted using a total RNA extraction kit (Tiangen Biochemical Technology Co., Ltd., Beijing, China), and cDNA was obtained using a reverse transcription kit (TIANScript cDNA, Tiangen Biotech Co., Ltd., Beijing, China), followed by real-time quantitative PCR reaction. The primers were provided by Sangon Biotech (Shanghai, China). The primers for FABP4 were 5'GAAATCACCGCAGACGACA-3' and 5'-TCATAACAC ATTCCACCACCA-3'. The primers for PTEN were 5'TTGAAGACCATAACCCACCA- $3^{\prime}$ and 5'-TACACCAG TCCGTCCCTTTC- $3^{\prime}$. The primers for GAPDH were $5^{\prime}$ CGGAGTCAACGGATTTGGTCGTAT-3' and 5'-AGC CTTCTCCATGGTGGTGA AGAC-3'. GAPDH was used as an internal reference for mRNA expression. PCR was conducted as follows: Pre-denaturation for $4 \mathrm{~min}$ at $94^{\circ} \mathrm{C}$, 1 cycle; denaturation for $30 \mathrm{~s}$ at $94^{\circ} \mathrm{C}$, annealing for $30 \mathrm{~s}$ at (56-62) ${ }^{\circ} \mathrm{C}$, extending for $1 \mathrm{~min}$ at $70{ }^{\circ} \mathrm{C}, 30-40$ cycles; and extra-extension for $5 \mathrm{~min}$ at $72^{\circ} \mathrm{C}$. The dissolution profile was analyzed, and the relative mRNA expression of FABP4 and PTEN was calculated by the $2^{-\Delta \Delta C T}$ method.

\section{MTT Assay}

3T3-L1 cells were seeded in 96-well culture plates at an initial density of $1 \times 10^{4}$ cells/well. After an overnight incubation, MTT solution (to a final concentration of $1 \mathrm{mg} / \mathrm{ml}$ ) was added. After $4 \mathrm{~h}$ of incubation in a $37^{\circ} \mathrm{C}$ incubator, $150 \mu \mathrm{L}$ dimethyl Sulfoxide (DMSO) was added into each well and cultured in a shaker for approximately $10 \mathrm{~min}$. Finally, the OD value at $490 \mathrm{~nm}$ wavelength was measured on microplate reader. The cell-free wells were used as the blank control. Survival rate $(\%)=(\mathrm{OD}$ value of the experiment group/OD value of the control group) $\times 100 \%$.

\section{Western Blot}

The collected cells were treated with cold RIPA buffer (Beyotime Institute of Biotechnology, Shanghai, China). After centrifuged, the supernatant was collected. The BCA kit (Thermo Fisher Scientific (China) Co., Ltd.) was used for protein quantification. A total of $50 \mu \mathrm{g}$ protein was separated by SDS-PAGE (Biyuntian Biotechnology Co., Ltd.) electrophoresis and transferred to nitrocellulose membrane (Pall Co., NY, USA). The membrane was blocked with 5\% skim milk at room temperature for 1 h. The antibodies including anti-FABP4 (1:200,
(Proteintech Group)), anti-PTEN (1:1000, (Proteintech Group)), internal reference Tubulin (1:200, (Proteintech Group)) and anti-GAPDH were added and cultured overnight at $4{ }^{\circ} \mathrm{C}$. Then, the diluted secondary antibody (Cell Signaling) was added and incubated for $1 \mathrm{~h}$ at room temperature. X-ray films were taken out in the dark chamber. Molecular Imager Chemi Doc XRS (BIO-RAD Co., California, USA) and JS-780 automatic gel imaging analysis systems were used for blotting and quantitative analysis. GAPDH was used as the internal control.

\section{Statistical Analysis}

All experiments were repeated three times with the same sample. Statistical analysis was made by software SPSS17.0 (International Business Machines, Corp., Armonk, NY, USA). Significant differences between groups were assessed by one-way analysis of variance (ANOVA). All data were expressed as means \pm standard deviation (SD). Differences were considered statistically significant when $P<0.05$.

\section{Results}

\section{The Differentiation and Characterization of 3T3LI Cells}

After inoculation for $16 \mathrm{~h}, 3 \mathrm{~T} 3-\mathrm{Ll}$ cells can basically achieve complete attachment (Figure 1A). It showed that the appearance was similar to that of fibroblasts, and the cell volume was smaller than that of primary cultured human preadipocytes. 3T3-Ll cells were spindle-shaped or polygon with multiple prominences. The nucleuses were round, locating in the center of the cytoplasm.

Microscopical observation was started on day 2 of differentiation induction for 3T3-Ll cells by switching to differential medium. It was noted that the cell volume was enlarged with local shuttle-shaped distention, and the distention increasingly grew bigger over differentiation time even with multiple distention in a cell. On day 4 of differentiation, reflective lipid droplets could be found in cells. With the progress of differentiation, more and more cells showed lipid droplets. Moreover, the number of lipid droplets in a single cell was gradually increasing, with the cell shape changed from polygon to oval or round. On day 8 of differentiation, the lipid droplets in some differentiated adipocytes merged together to form larger ones, which pushed the nucleus towards one side of the cell. Oil red O staining can specifically color the lipid in differentiated adipocytes red (Figure 1B). 


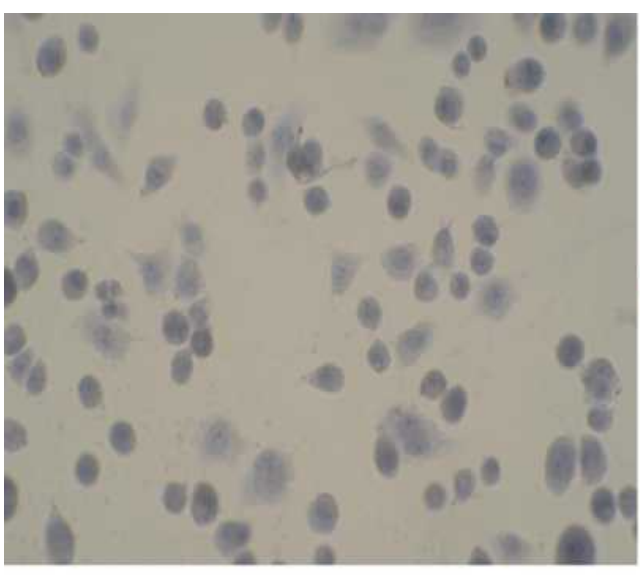

A

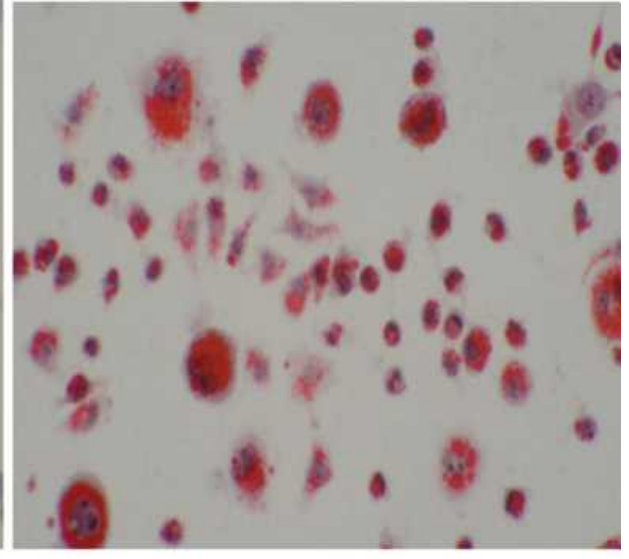

B

Figure I The differentiation and characterization of 3T3-LI cells. (A) The cell morphology of 3T3-LI cells without induction. (B) The differentiation of 3T3-LI cells on day 8 of induction. Images ( $40 \times$ magnification) are representative images of 3 independent samples.

\section{The A-FABP mRNA Expression Level After siRNA Different Types of A-FABP}

siRNA is a double-stranded RNA molecule with 21-25 nucleotides. It can trigger the silence of target mRNA which is mutually complementary to it. We used three types of siRNA to inhibit the A-FABP mRNA expression level. During the differentiation process of 3T3-L1 cells, the siRNA with the highest inhibition efficiency was utilized to investigate the action and potential mechanism of A-FABP. qPCR was used to detect the A-FABP mRNA expression level in 3T3-L1 cells. As shown in Figure 2, compared to the negative control group, the A-FABP mRNA expression level in the siRNA1, siRNA2 and siRNA3 groups was significantly decreased $(P<0.05)$. Moreover, siRNA3 notably inhibited the A-FABP mRNA expression level in comparison with siRNA2 and siRNA3 groups. Therefore, siRNA3 was used to inhibit the A-FABP mRNA expression for further experiment.

\section{Inhibition of A-FABP or Induction with Insulin Significantly Decreased the Proliferation in 3T3-LI Cells}

As shown in Figure 3, the proliferation in 3T3-L1 cells was significantly reduced on day 6 after induction with insulin and dexamethasone $(P<0.05)$. Regardless of with or without induction, inhibition against the A-FABP expression could increase the proliferation activity $(P<$

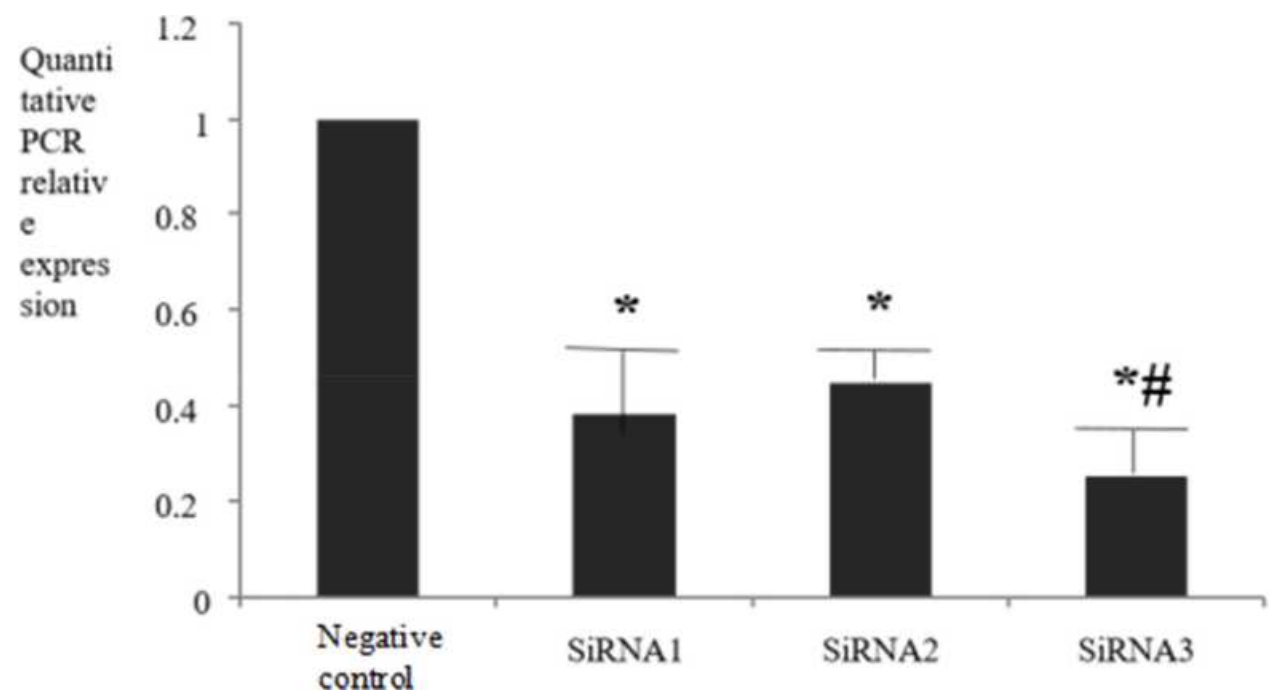

Figure 2 The A-FABP mRNA expression level after transferred with siRNAI, siRNA2 or siRNA3. $* P<0.05$ compared with the negative group; ${ }^{*} P<0.05$ compared with the SIRNAI or SIRNA2 group. 


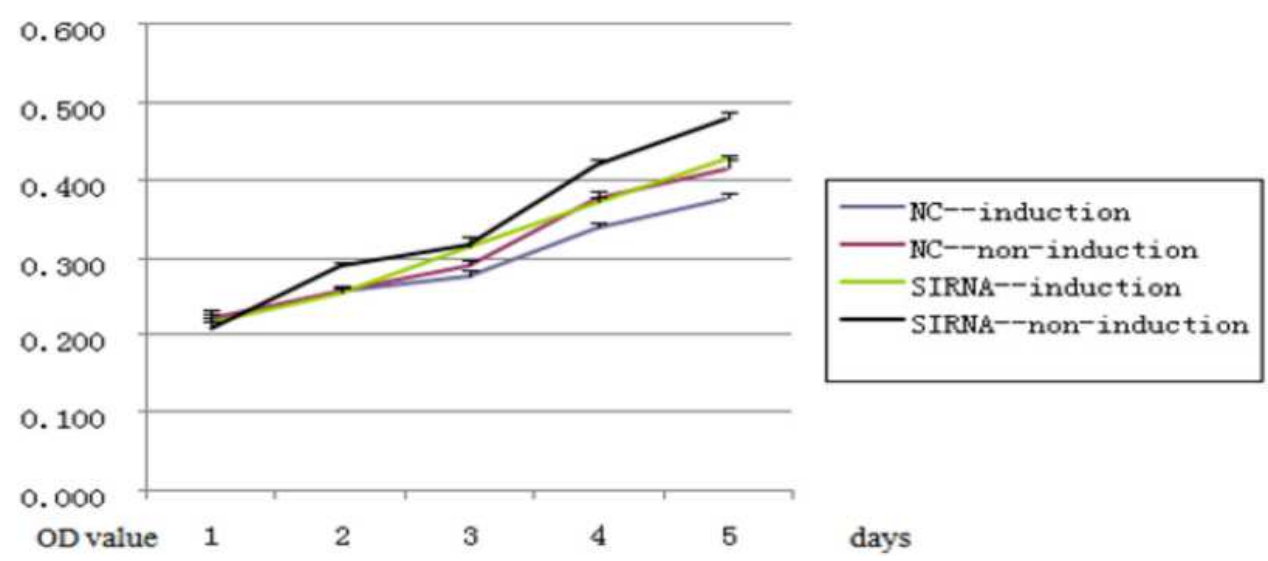

Figure 3 Growth curves of the 3T3-LI cells after inhibition of A-FABP or induction with insulin.

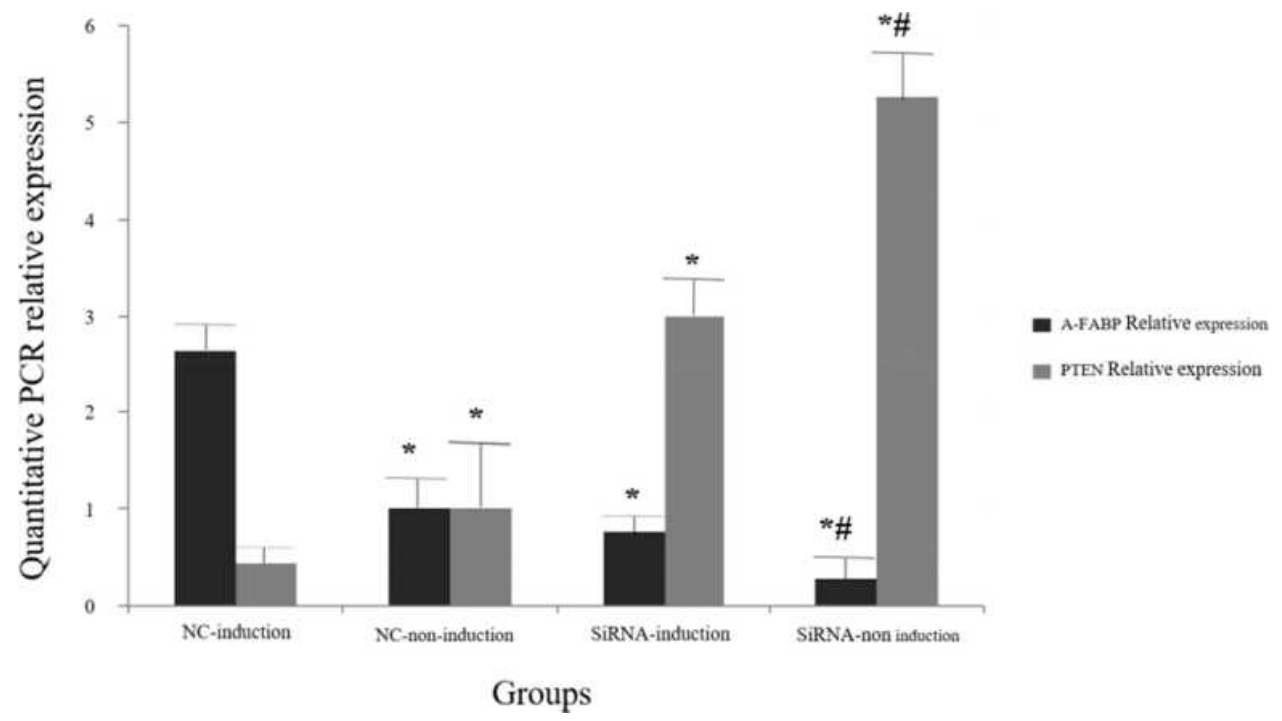

Figure 4 The mRNA expression levels of A-FABP and PTEN after inhibition of A-FABP or induction with insulin. $* P<0.05$ compared with the induction group; ${ }^{\#} P<0.05$ compared with the siRNA + induction group.

$0.05)$. The increased proliferation activity started from day 2 in the non-induction group, and started from day 4 in the induction group. For the group with the inhibition of A-FABP, the proliferation activity of cells was reduced after induction, which started from day $2(P<0.05)$.

\section{The mRNA Expression Levels of A-FABP and PTEN After Inhibition of A-FABP or Induction with Insulin}

As shown in Figure 4, compared with the non-induction group, the A-FABP mRNA expression in the 3T3-L1 cells was significantly increased after induction by 2.62 times, and the PTEN mRNA expression was notably decreased by 0.45 times. Moreover, interfering the A-FABP expression in the induced 3T3-L1 cells could increase the PTEN mRNA expression by 6.4 times. Similarly, interfering A-FABP expression in the 3T3-L1 cells could also increase the PTEN mRNA expression by 5.26 times.

The Protein Expression Levels of A-FABP, $\mathrm{P}-\mathrm{AKT}$ and PTEN After Inhibition of A-FABP or Induction with Insulin

As shown in Figure 5, during the proliferation process of 3T3-L1 cells, the protein expression levels of A-FABP, p-AKT and PTEN between the siRNA interference and non-interference groups did not change significantly. Compared to the non-induction group, the protein expression levels of A-FABP and p-AKT were significantly 


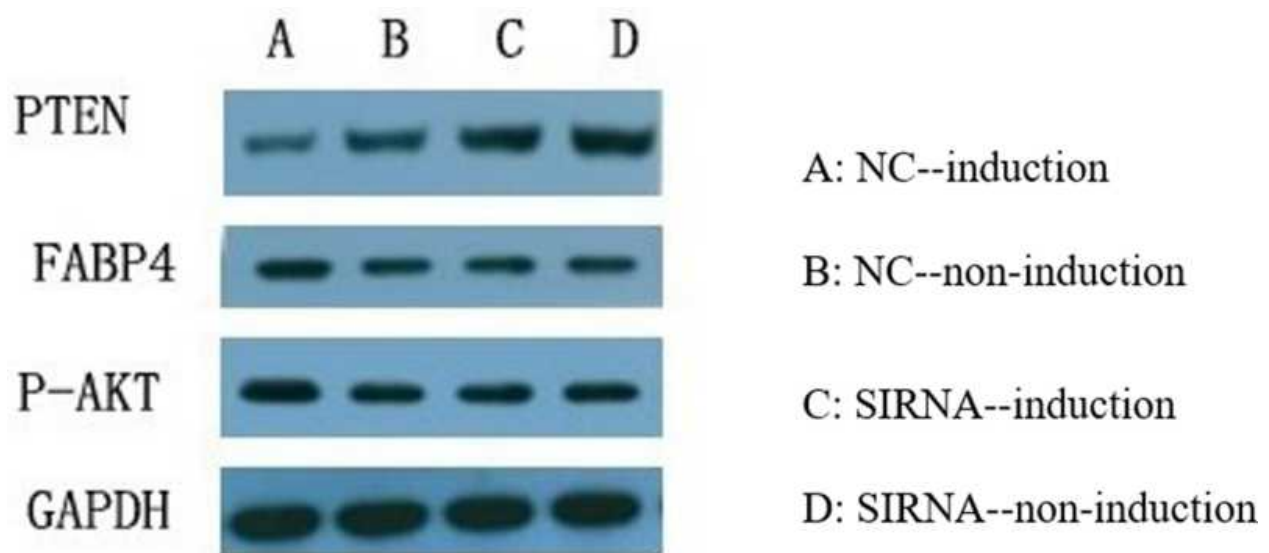

Figure 5 The protein expression levels of A-FABP, p-AKT and PTEN after inhibition of A-FABP or induction with insulin.

increased in the induction group. However, the PTEN protein expression level in the induction group was significantly decreased in comparison with the non-induction group. Furthermore, the A-FABP protein expression level was markedly decreased in the siRNA groups in comparison with the control groups. The PTEN protein expression was significantly increased in the siRNA groups, but the p-AKT protein expression was markedly decreased in comparison with the control induction group.

\section{Discussion}

Obesity is a consequence of excessive growth of fat tissues, and the growth of fat tissues includes the increase of adipocytes as well as the formation of new adipocytes differentiated from precursor cells. The contribution of A-FABP in fat tissues is still unknown. We investigated the changes of cell proliferation activity in both induction and non-induction groups after interfered the A-FABP expression of preadipocytes. In both induction and noninduction groups, inhibition of A-FABP increased cell proliferation activity, indicating that A-FABP played an important role in the induction of differentiation and maturity of preadipocytes. It is already known that the occurrence of obesity is closely associated with the growth of adipocytes. It has been reported that $15-50 \%$ of fat tissues is composed of preadipocytes, which can divide, differentiate and even die under the stimulation of extracellular signals. ${ }^{2}$ Therefore, it can be seen that controlling the proliferation of preadipocytes as well as inducing apoptosis of preadipocytes has an important role in obesity prevention. As mature adipocytes do not divide anymore, its population mainly depends on the proliferation and differentiation of preadipocytes as well as the apoptosis. ${ }^{1}$
Inducing apoptosis of mature adipocytes is believed to be a significant way to control obesity.

In recent years, with the gradual understanding of the role of adipose tissue as an endocrine organ, people have found that endocrine dysfunction in fat tissues is an important bridge between obesity and DM. While abnormal expression of adipocyte-derived factors is an important molecular mechanism to participate or aggravate IR as well as cell function impairment, and consequently to induce DM. The expression disorder of fat-derived factors plays an important role in the pathophysiology process of DM caused by inflammation. A number of studies have showed that AKT cascade signal response plays a significant role in the formation of adipocytes. Under physiological status, insulin binds to the $\alpha$ subunit of its receptor, thus induces the autophosphorylation of tyrosine residue of $\beta$ subunit, and activates the phosphorylation of tyrosine kinase of insulin receptor. PI-3K, a lipid kinase, can catalyze the phosphorylation of phosphatidylinositol on cell membrane and produce phosphatidyl inositol triphosphate that will then bind to downstream signal molecules. ${ }^{16}$ The downstream signal molecules of PI3K is a 3-phosphatidylinositol-dependent protein kinase and AKT. AKT could be activated by 3-phosphatidylinositol-dependent protein kinase to facilitate the migration of intracellular GLUT4 to cell membrane.

The expression and activity of A-FABP are significantly enhanced during the differentiation process of adipocytes. Moreover, A-FABP plays a critical role in insulin resistance induce by obesity. Our study has demonstrated that the A-FABP mRNA expression is increased after induced differentiation of 3T3-L1 cells, while the PTEN expression is considerably reduced. Interfering the A-FABP mRNA expression during the induction of 3T3-L1 cells is able to 
increase the PTEN mRNA expression. However, interfering the A-FABP mRNA expression during the proliferation of 3T3-L1 cells is able to significantly increase the PTEN mRNA expression. Protein-level investigation in this experiment has suggested that interfering the A-FABP protein expression during the proliferation of 3T3-L1 cells does not result in any apparent change. The reason is considered to be related to the fact that induced differentiation is a complex physiological process and A-FABP protein expression is limited during the proliferation of undifferentiated 3T3-L1 cells. During the differentiation of 3T3-L1 cells, siRNA's interference to A-FABP expression decreases the p-AKT protein expression and also increases the content of PTEN protein. It is considered that A-FABP may participate in insulin resistance. However, whether it directly acts on p-AKT or indirectly acts on PTEN in insulin signaling pathway needs further investigation.

This study has proved that interfering A-FABP expression during the differentiation of normal adipocytes can reduce the p-AKT protein expression to disturb carbohydrate metabolism. Previous study has found that high AKT protein expression is an important factor for adipocytes to maintain its normal physiology and refrain from apoptosis. Our results showed that A-FABP expression is essential during the normal differentiation of adipocytes. Contrary to our study, previous animal experiments have indicated that inhibiting A-FABP expression can improve insulin resistance. The reason may be the fact that compared with normally differentiated adipocytes, abnormally differentiated adipocytes have abnormalities in the A-FABP expression, or inhibition of A-FABP expression gives rise to reduced p-AKT level. Therefore, it improves insulin resistance. However, the specific mechanism needs further study.

\section{Conclusion}

At cellular level, this study has revealed that interfering A-FABP expression during the differentiation of normal adipocytes can reduce $\mathrm{p}-\mathrm{AKT}$ expression to disturb carbohydrate metabolism. This study prompts that A-FABP may participate in the molecular mechanism of insulin resistance, which lays the foundation for further investigations on insulin-resistant adipocytes.

\section{Data Sharing Statement}

The datasets used and/or analyzed during the current study are available from the Jian Huangfu and Rui Xiao on reasonable request.

\section{Ethics Approval and Consent to Participate}

The current study was approved by the Animal Ethics Committee and was conducted in accordance to the relevant agreements with The Affiliated Hospital of Inner Mongolia Medical University. All procedures were performed in accordance with the Guidance Suggestions for the Care and Use of Laboratory Animals, formulated by the Ministry of Science and Technology of China.

\section{Consent for Publication}

Not applicable.

\section{Author Contributions}

All authors made substantial contributions to conception and design, acquisition of data, or analysis and interpretation of data; took part in drafting the article or revising it critically for important intellectual content; agreed to submit to the current journal; gave final approval of the version to be published; and agree to be accountable for all aspects of the work.

\section{Funding}

We thank for the financial support from Natural Science Foundation Project Plan of Inner Mongolia Autonomous Region(2019MS08160).

\section{Disclosure}

The authors state that there are no financial, personal, or professional conflicts of interests that may hinder this work.

\section{References}

1. Hammarstedt A, Gogg S, Hedjazifar S, et al. Impaired adipogenesis and dysfunctional adipose tissue in human hypertrophic obesity. Physiol Rev. 2018;98(4):1911-1941. doi:10.1152/physrev.00034.2017

2. Yongmei L, Jianying D, Zhu E, et al. Liraglutide suppresses proliferation and induces adipogenic differentiation of 3T3-L1 cells via the hippo-YAP signaling pathway. Mol Med Rep. 2018;17(3):4499-4507. doi:10.3892/mmr.2018.8438

3. Furuhashi M. Fatty acid-binding protein 4 in cardiovascular and metabolic diseases. $J$ Atheroscler Thromb. 2019;26(3):216-232. doi: $10.5551 /$ jat. 48710

4. Ghallab A, Seddek A. PPARG as therapeutic target for antifibrotic therapy. EXCLI J. 2020;19:227-229. doi:10.17179/excli2020-1136

5. Yang R, Castriota G, Chen Y, et al. RNAi-mediated germline knockdown of FABP4 increases body weight but does not improve the deranged nutrient metabolism of diet-induced obese mice. Int $J$ Obes (Lond). 2011;35(2):217-225. doi:10.1038/ijo.2010.128

6. Floresta G, Pistarà V, Amata E, et al. Adipocyte fatty acid binding protein 4 (FABP4) inhibitors. a comprehensive systematic review. Eur $J \quad$ Med Chem. 2017;138:854-873. doi:10.1016/j.ejmech.201 7.07 .022 
7. Xu A, Wang Y, Xu YJ, et al. Adipocyte fatty acid-binding protein is a plasma biomarker closely associated with obesity and metabolic syndrome. Clin Chem. 2006;52(3):405-413. doi:10.1373/clinche m.2005.062463

8. Hopkins BD, Hodakoski C, Barrows D, Mense SM, Parsons RE. PTEN function: the long and the short of it. Trends Biochem Sci. 2014;39(4):183-190. doi:10.1016/j.tibs.2014.02.006

9. Gorbenko O, Panayotou G. Identification of novel PTEN-binding partners: PTEN interaction with fatty acid binding protein FABP4. Mol Cell Biochem. 2010;337(1-2):299-305. doi:10.1007/s11010009-0312-1

10. Tsuda M, Inoue-Narita T, Suzuki A, et al. Induction of gene encoding FABP4 in Pten-null keratinocytes. FEBS Lett. 2009;583 (8):1319-1322. doi:10.1016/j.febslet.2009.03.030

11. Yuan-yuan LI, Rui XIAO, Jiang-feng MAO, et al. Increased plasma levels of FABP4 and PTEN Is associated with more severe insulin resistance in women with gestational diabetes mellitus. Med Sci Monit. 2015;21:426-431. doi:10.12659/MSM.892431
12. Su D, Zhang C-L, Jian H-F, et al. Gene expression and correlation of Pten and Fabp4 in liver, muscle, and adipose tissues of type 2 diabetes rats. Med Sci Monit. 2015;21:3616-3621. doi:10.12659/ MSM.895490

13. Ngeow J, Eng C. PTEN in hereditary and sporadic cancer. Cold Spring Harb Perspect Med. 2020;10(4):a036087. doi:10.1101/cshperspect.a036087

14. Yang J, Yin Y. PTEN in chromatin remodeling. Cold Spring Harb Perspect Med. 2020;10(2):a036160. doi:10.1101/cshperspect. a036160

15. Glenn RM, Roger LW. Structural mechanisms of PTEN regulation. Cold Spring Harb Perspect Med. 2020;10(3):a036152. doi:10.1101/ cshperspect.a036152

16. Deng T, Wang Y, Wang C, et al. FABP4 silencing ameliorates hypoxia reoxygenation injury through the attenuation of endoplasmic reticulum stress-mediated apoptosis by activating PI3K/Akt Pathway. Life Sci. 2019;224:149-156. doi:10.1016/j.1fs.2019.03.046

\section{Publish your work in this journal}

Diabetes, Metabolic Syndrome and Obesity: Targets and Therapy is an international, peer-reviewed open-access journal committed to the rapid publication of the latest laboratory and clinical findings in the fields of diabetes, metabolic syndrome and obesity research. Original research, review, case reports, hypothesis formation, expert opinion and commentaries are all considered for publication. The manuscript management system is completely online and includes a very quick and fair peer-review system, which is all easy to use. Visit http://www.dovepress.com/testimonials.php to read real quotes from published authors. 